\title{
EVALUATION OF SOLDIER INTEGRATED PROTECTIVE ENSEMBLES USING ANALYTIC HIERARCHY PROCESS
}

\author{
Refik Soyer \\ The George Washington University \\ and \\ Raymond Spring \\ The US Army Natick Research, Development \& Engineering Center
}

\begin{abstract}
In this paper, we present a multi-attribute evaluation model for Soldier Integrated Protective Ensemble (SIPE) modules using Analytic Hierarchy Process. Evaluation of SIPE modules requires consideration of multiple factors associated with performance, survivability and cost issues. We present a model that takes into account relevant factors in SIPE evaluation. We emphasize the model building aspects and discuss how the model is structured to include various qualitative and quantitative factors. We also present an example of evaluation using the currently used protective systems.
\end{abstract}

\section{INTRODUCTION}

As described in SIPE Advanced Technology Transition Demonstration (ATTD) Tecnology Development Plan 1990 -henceforth ATTD Plan (1990)-, SIPE is a "modular head-to-toe individual fighting system which will allow the soldier improved combat effectiveness while providing improved survivability against multiple battlefield hazards." As pointed out in ATTD Plan (1990), the current protective clothing and equipment require separate units to provide protection from different weapon hazards. Such protection systems overburden the soldier with excess weight, interfere with soldier's ability to use weapons, affect communication and therefore result in degradation of overall performance of the soldier.

In consideration of the above, a program has been initiated to develop SIPE modules that will eliminate redundancies of the existing systems, to provide protection and to improve the performance of the individual soldier. Once, such modules are developed, an important issue is how to evalute them. Evaluation of SIPE modules requires consideration of multiple factors associated with performance, survivability and cost issues and therefore necessitates use of a multi-attribute evaluation technique such as the Analytic Hierarchy 
Process (AHP) [see Saaty (1980)]. Since the individual SIPE -modules have not been developed yet, in this paper we use a top-down approach to structure the evaluation model. AHP and its microcomputer software adaptation, Expert Choice (EC) [see Forman et. al. (1983)] enable us structure the complexity in an hierarchical manner and operationalize the evaluation process.

In Section II, we present a model that takes into account the relevant factors in SIPE evaluation. We discuss all these factors and structure our model using EC. In Section III, we give an example of evaluation by applying the model to currently used protection systems in U.S. Army. We note that the emphasis of this paper is on the model building aspects of the SIPE evaluation problem since the actual SIPE modules have not been developed yet.

\section{SIPE EVALUATION MODEL}

The examination of possible SIPE candidates should be made in the context of how a particular module will contribute to achievement of the objectives of SIPE concept. In what follows we present an EC model which takes into account the relevant factors in evaluation of SIPE modules.

The EC model is shown as a value tree in Figure 1. The first level of the model involves four main evaluation criteria. Some of these criteria have subcriteria and possibly even sub-subcriteria or attributes. The four main criteria considered are: Protection, Performance, Cost and Synergy; these will be discussed in the following sections. The lowest level of the model consists of the candidate SIPE modules which will be evaluated with respect to the criteria, subcriteria, or sub-subcriteria. For illustrative purposes we consider three protection systems that are currently used by soldiers. These are: Battlefield Dress Uniform (BDU), Personnel Armor System for Ground Troops (PASGT) and Mission Oriented Protective Posture (MOPP).

In Figure 2, we present the first level of the hierarchy, that is the main four criteria. We note that the derived priorities imply that Protection and Performance are the two more important criteria in the evaluation. The description of the each criterion and the associated sub-criteria are given next.

\subsection{Protection Considerations:}

This main criterion allows the evaluation of the SIPE modules with respect to protection provided against various military hazards. The subcriteria considered under protection are: protection against climate hazards (CLIMATE in the model), ballistic weapons (BALIISTI), nuclear weapons (NUCLEAR), chemical and biological weapons (CHEM/BIO), directed energy weapons (DEW/LAS), and against flame weapons (FLAME). The final subcriterion considered is camouflage (CAMOUFLA) provided 
Figure 1: The Value Tree for the SIPE Evaluation Hodel.

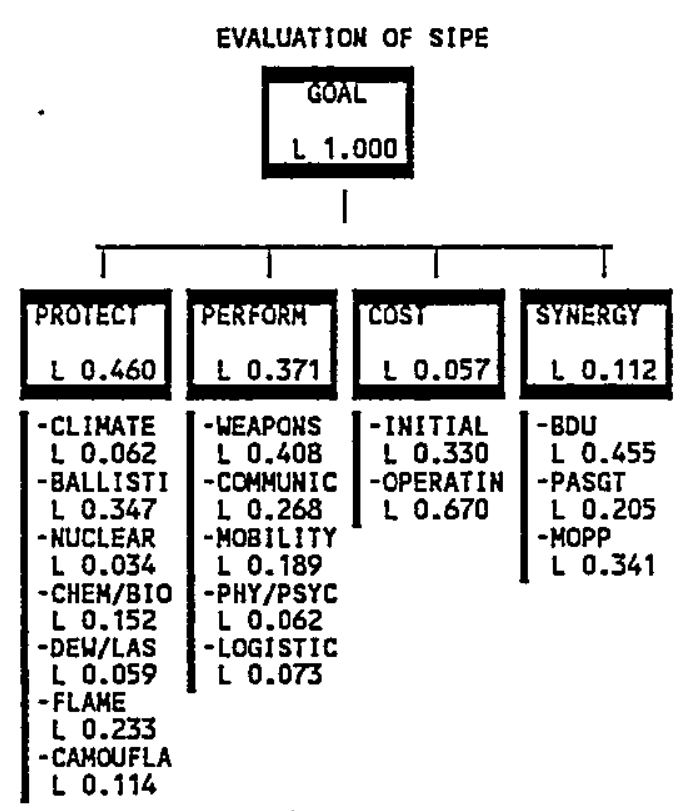

Figure 2: Main Criteria and Subcriteria for SIPE Evaluation. 
by the module. The protection criterion and its associated subcriteria are shown by the hierarchy in Figure 3. The priorities are derived via judgments using the survey of Sampson, Tucker and Ridgeway (1989).

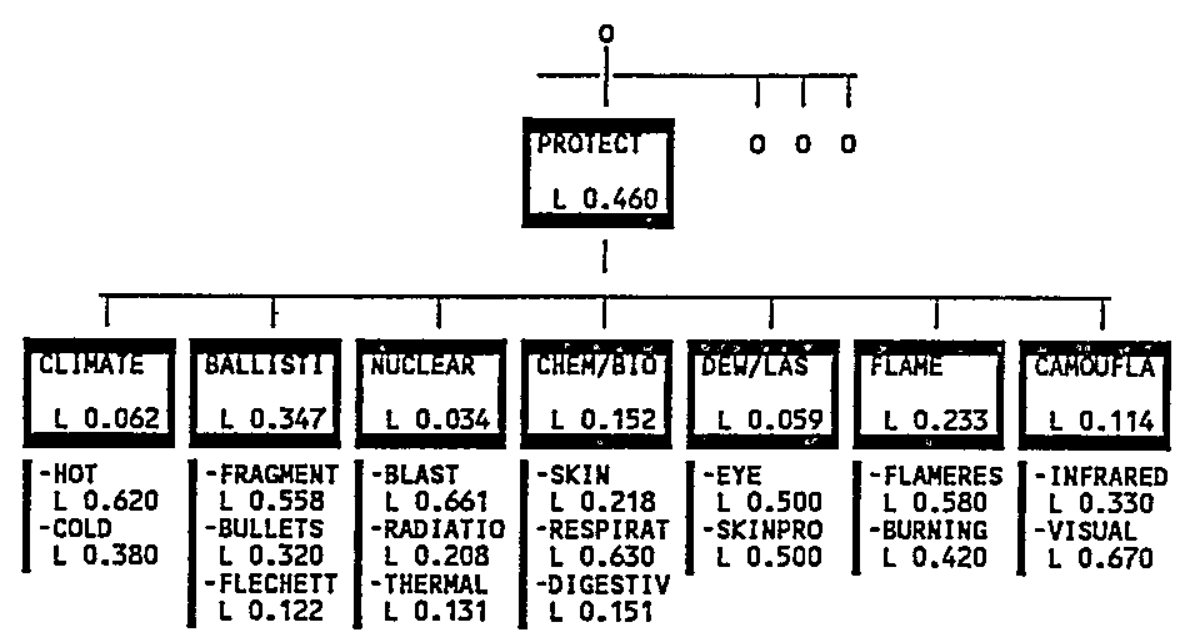

Figure 3: Protection Hierarchy and Subcriteria.

2.1.1 Climatic Protection: Protection against climatic hazards (CLIMATE) refers to protection provided by a module against both excessive heat and cold. The hierarchy corresponding to climatic protection is shown in Figure 4. This includes two subcriteria: protection against excessive heat (HOT) and protection against excessive cold (COLD). Protection against extreme heat can be achieved by minimizing heat load (HEATLOAD), providing transfer of body moisture (MOISTURE) and by increasing internal air movement (AIRMOVE). These three attributes are represented as the sub-subcriteria under heat protection and provide basis for evaluation of different SIPE modules. Similarly, protection against cold is achieved by minimizing radiant heat. loss (HEATLOSS), by preventing penetration of precipitation and wind (PENETRATE)and by allowing transfer of body moisture (MOISTURE). These three attributes are the sub-subcriteria of climatic protection in Figure 4.

2.1.2 Ballistic Protection: Ballistic protection (BALLISTI) refers to protection provided by SIPE module against conventional ballistic weapons and explosives such as bullets, fragments and flechettes. These weapons are the leading cause of injury and death in modern warfare. Figure 5 shows the ballistic protection hierarchy with subcriteria as protection against fragments (FRAGMENT), protection against bullets (BULLETS) and protection against flechettes. Under each of the subcriteria there are two attributes which refers to the level of head protection (HEAD) and body protection (BODY) provided by the SIPE module against each of these ballistic weapons.

2.1.3 Nuclear Protection: Nuclear protection (NUCLEAR) refers to protection against nuclear weapons that can be delivered by missiles, bombs and artillery. Nuclear wepons produce three major hazards: blast, heat and ionizing radiation. The hierarchy 

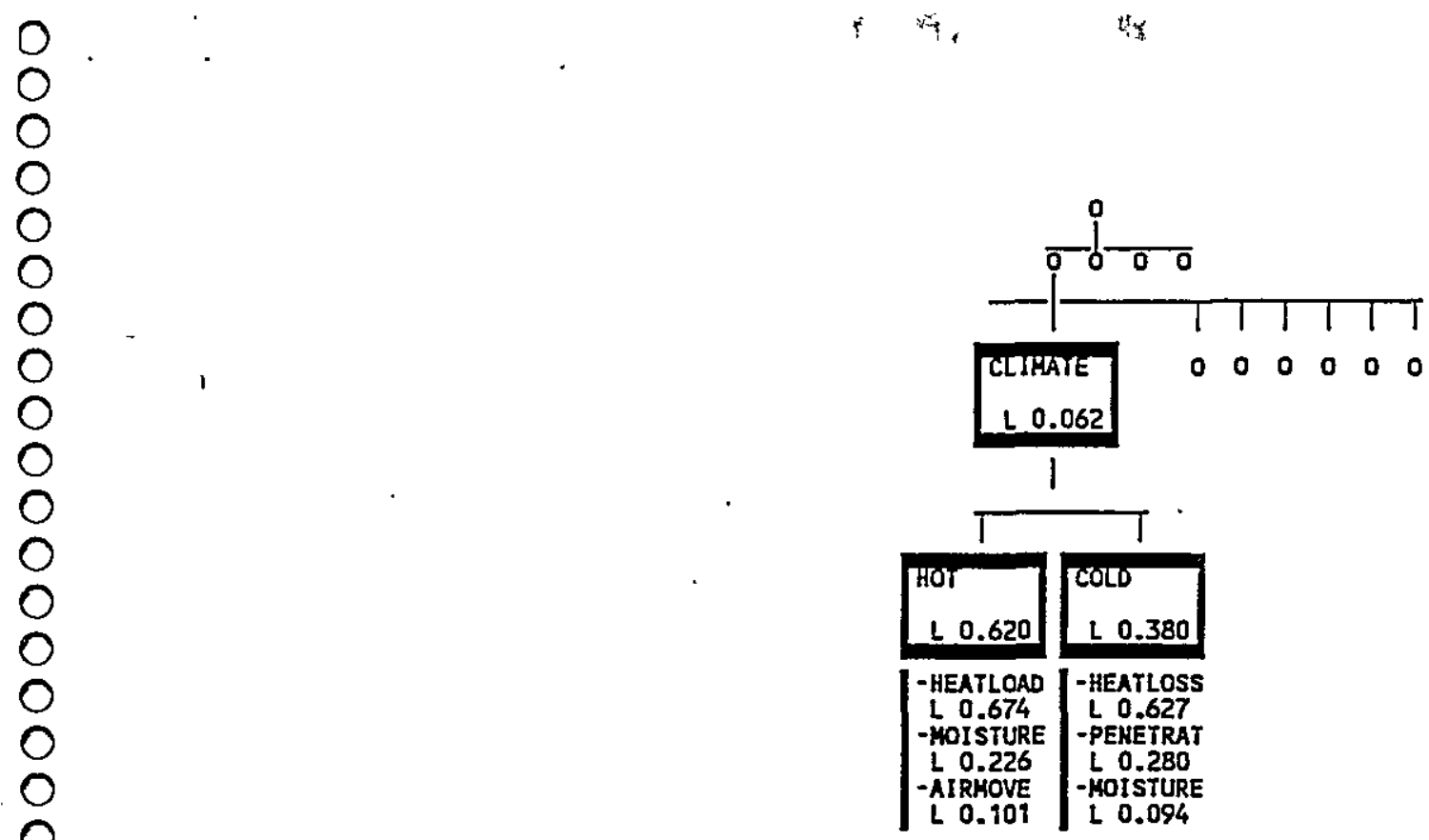

Figure 4: Climatic Protection Hierarchy.

associated with nuclear protection is shown in Figure 6. The three subcriteria represent protection against nuclear blast (BLAST), protection against residual radiation (RADIATIO) and protection against thermal (heat) radiation (THERMAL). Under residual radiation the two attributes refer to protection against early fallout (EARLY) and delayed fallout (DELAYED). We note that protection against initial radiation is not considered in the model since it is known that the highly penetrating character of initial radiation can not be prevented by any protective clothing [See Sampson et al. (1984)].

2.1.4 Chemical and Biological Protection: Chemical and biological protection (CHEM/BIO) refers to protection against chemical and biological weapons that can cause mass destruction. Since protection requirements against chemical and biological agents are similar, they are considered as a single factor in our model. The hierarchy associated with chemical and biological protection is given in Figure 7. The three subcriteria refer to level of skin protection (SKIN), protection of respiratory organs (RESPIRAT) and protection of digestive organs (DIGESTIV). That is, the three modules will be evaluated with respect to the level of protection they provide for skin, respiratory organs and digestive organs against chemical and biological agents.

2.1.5 Directed Energy Weapon Protection: In general protection against directed energy weapons (DEW/LAS) refers to protection against lasers. The hierarchy shown in Figure 8 contains two attributes that represent the level of eye protection (EYE) and the level skin protection (SKINPRO) provided by the protection system.

2.1.6 Flame Weapon Protection: Flame/Incendiary weapon protection (FLAME) refers to protection against weapons that uses flame agents (such as napalm) or incendiary agents (such as incendiary cluster bombs). Protection against such. weapons can be evaluated in terms of flame resistance of protective material (FLAMRES) and its resistance to burning (BURNING). These two attributes are presented as the subcriteria of the hierarchy shown in Figure 9. 


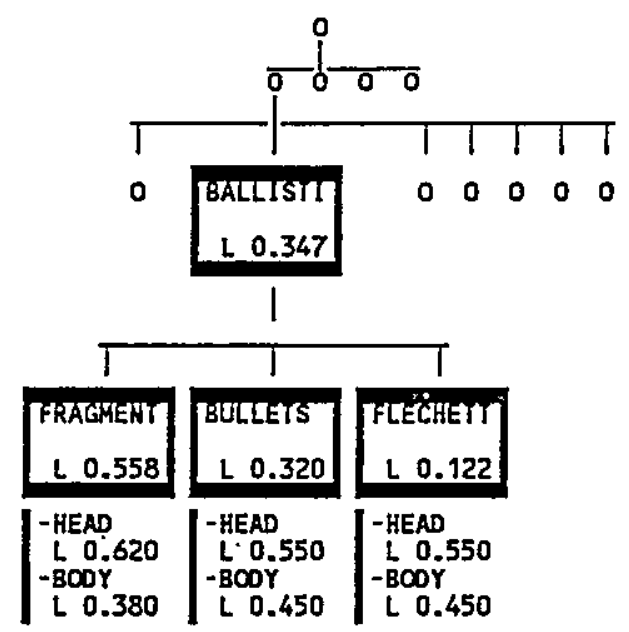

Figure 5: Ballistic Protection Hierarchy.

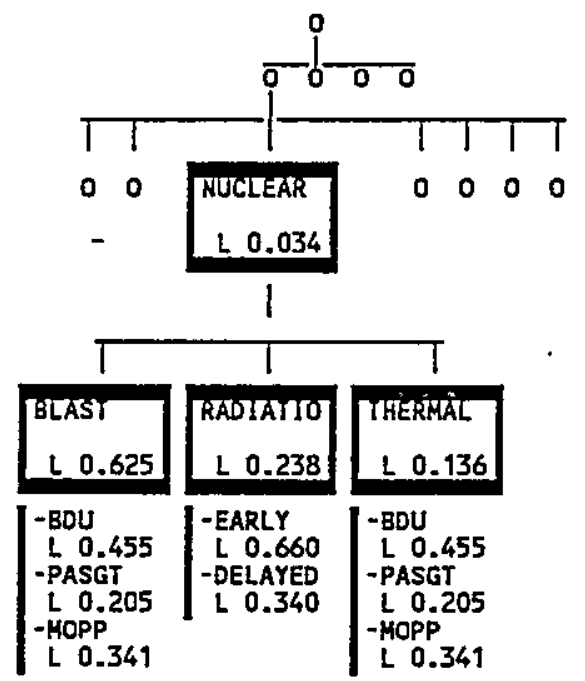

Figure 6: Nuclear Protection Hierarchy.
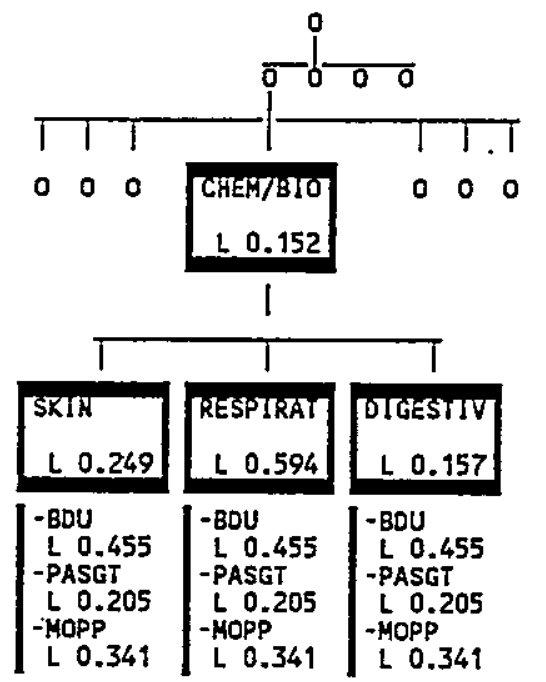

Figure 7: Hierarchy for Chemical and Biological Protection. 


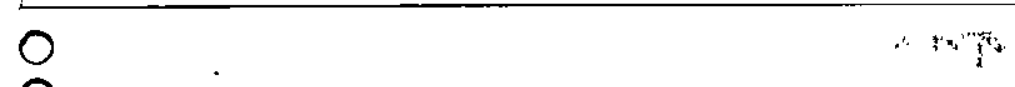

2.1.7 Camouflage Protection: Camouflage protection (CAMOUFLA) refers to a SIPE module's ability in decreasing soldier's detection by enemy forces and therefore soldier's vulnerability. This is usually defined in terms of camouflage against visual

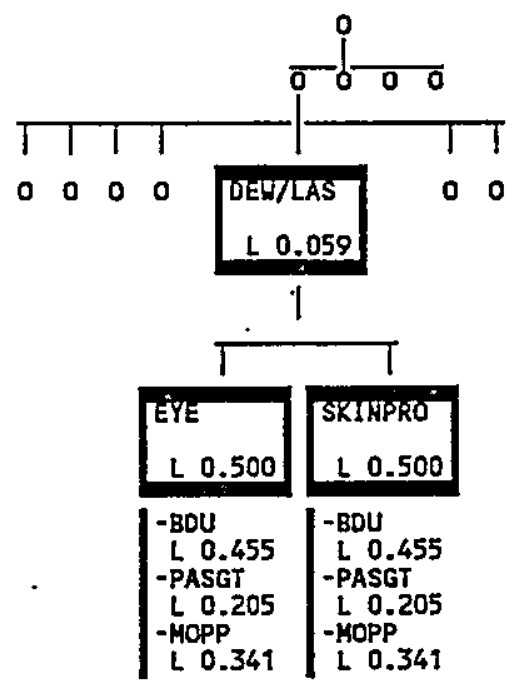

Figure 8: Hierarchy for Directed Energy Heapon Protection.
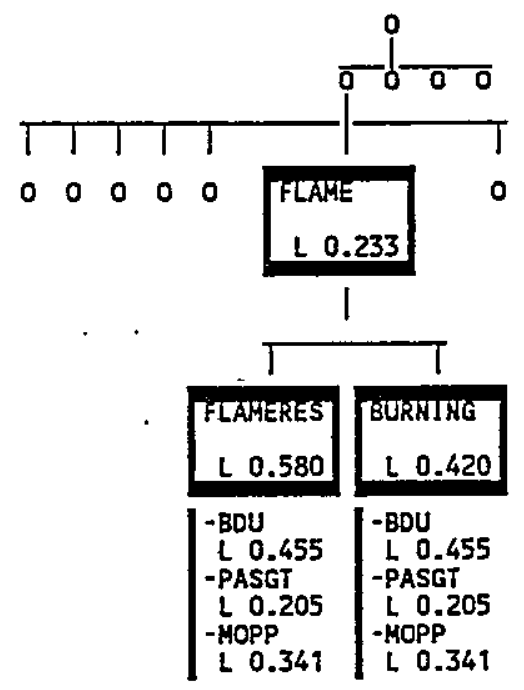

Figure 9: Hierarchy for $\mathrm{Flame}$ Weapons Protection.

detection (VISUAL) and camouflage against infrared detection devices (INFRARED). These two attributes are included as the subcriteria of the hierarchy in Figure 10.

\subsection{Performance Considerations:}

The second main criterion in Figure 2 involves the effect of a SIPE module on soldier's performance (PERFORM). A SIPE module is preferable to the extent that it minimizes performance degradation due to increased protective equipment. Soldier's evaluating the effect of a SIPE module on soldier's performance. Figure 11 shows the performance hierarchy and the associated subcriteria. performance is affected by technical 


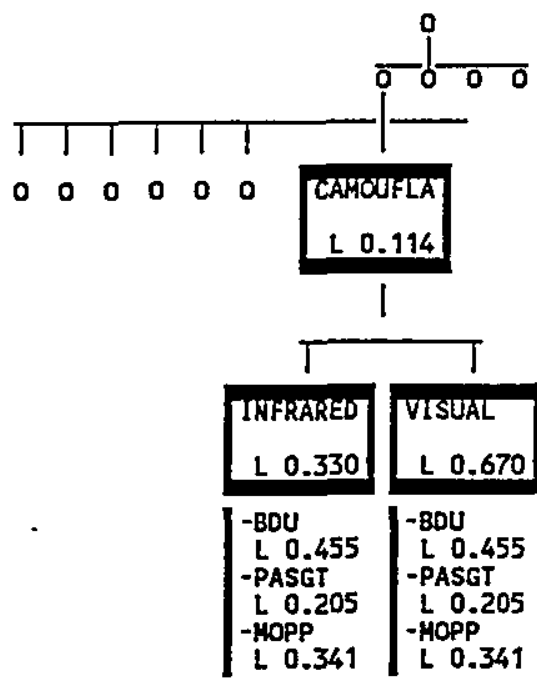

Figure 10: Hierarchy for Camouftage Considerations.

components such as the Weapons interface (WEAPONS) and Communication equipment (COMMUNIC) provided by a SIPE module. Furthermore, Physiological and Psychological factors (PHY/PSYC), Soldier's level of Mobility (MOBILITY) and Logistics considerations

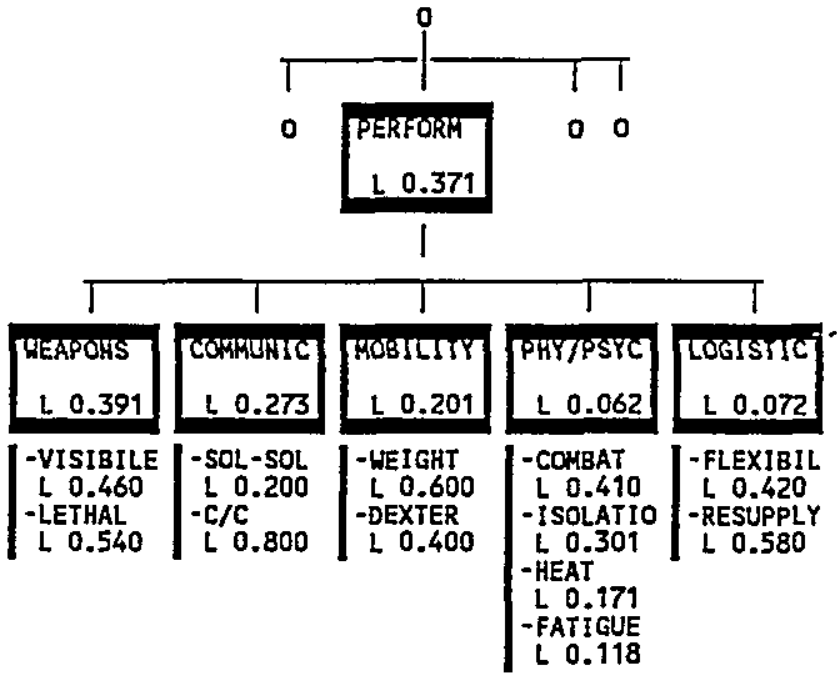

Figure il: Performance Hierarchy and Subcriteria.

(LOGISTIC) are all relevant in evaluating the effect of a module on soldier's performance. Figure 11 shows the performance hierarchy and associated subcriteria.

2.2.1 Weapons Interface: This factor evaluates a module with respect to its capabilities in terms of identifying targets during limited visibility (VISIBLE) and in terms of its effects on lethality (LETHAL). These two factors are considered as the subcriteria of the hierarchy shown in Figure 12. Under VISIBLE there are two sub-subcriteria which represent the effects on all weather visibility (WEATHER) and day/night capability (DAY/NITE) respectively. 


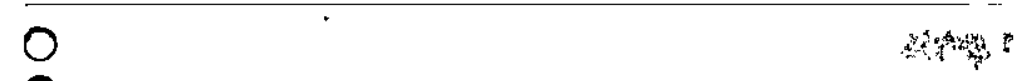

2.2.2 Communication: The effect of a SIPE module on soldier communication can be evaluated in terms of two subcriteria: Soldier-to-Soldier Communication (SOL-SOL), and Command/Control Communication $(\mathrm{C} / \mathrm{C})$ which are shown in Figure 13.

2.2.3 Mobility: This criterion evaluates a SIPE module with respect to its effect on soldier's bulk/weight (WEIGHT) and on soldier's manual dexterity (DEXTER) which are included as the subcriteria in Figure 14.

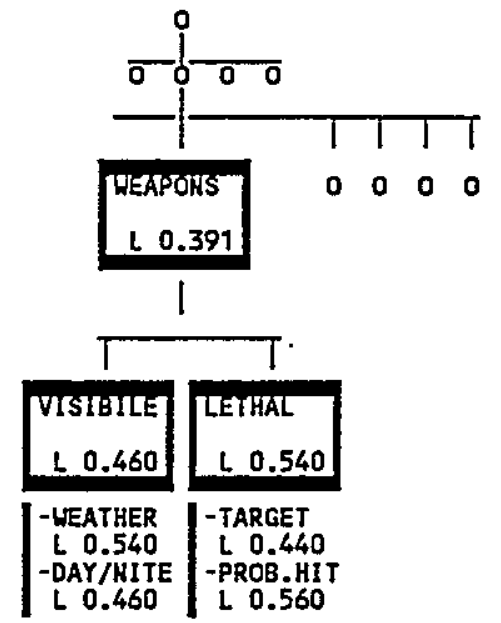

Figure 12: Hierarchy for Weapons Interface.

2.2.4 Physiological/Psychological Factors: This criterion involves the physiological/psychological effects of a SIPE module in terms of its contribution to combat anxiety (COMBAT), isolation stress (ISOLATIO), heat stress (HEAT) and soldier's fatigue (FATIGUE). The four criteria are shown in Figure 15.

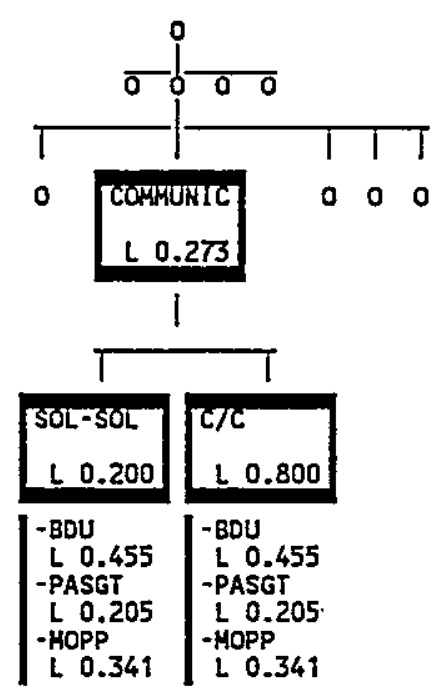

Figure 13: Communication Hierarchy. 
2.2.5 Logistics Concerns: This factor considers the effect of a SIPE module on the soldier's flexibility (FLEXIBIL) in terms of adaptation to these tasks and the resupply needs (RESUPPLY) to achieve the tasks. The Hierarchy is shown in Figure 16.

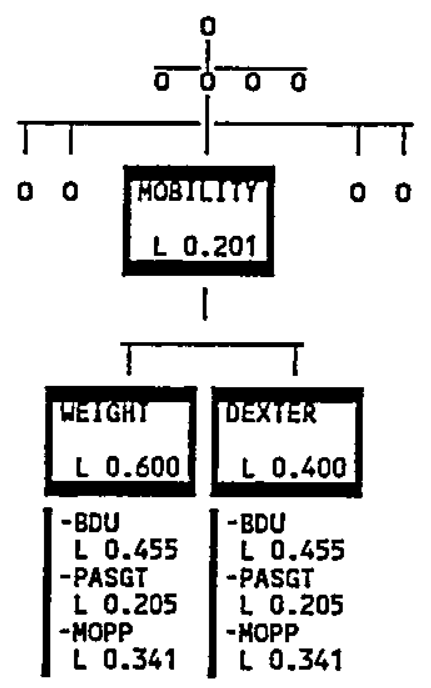

Figure 14: Hierarchy for Mobility Considerations.

\subsection{Cost Considerations:}

The third main criterion in Figure 2 considers the costs (COST) associated with a SIPE module. The cost hierarchy shown in Figure 17 includes initial costs (INITIAL) associated with development of the module and the operating costs (OPERATIN). The operating costs include maintenance costs (MAINT), training costs (TRAIN) and repair costs (REPAIR) associated with a module.

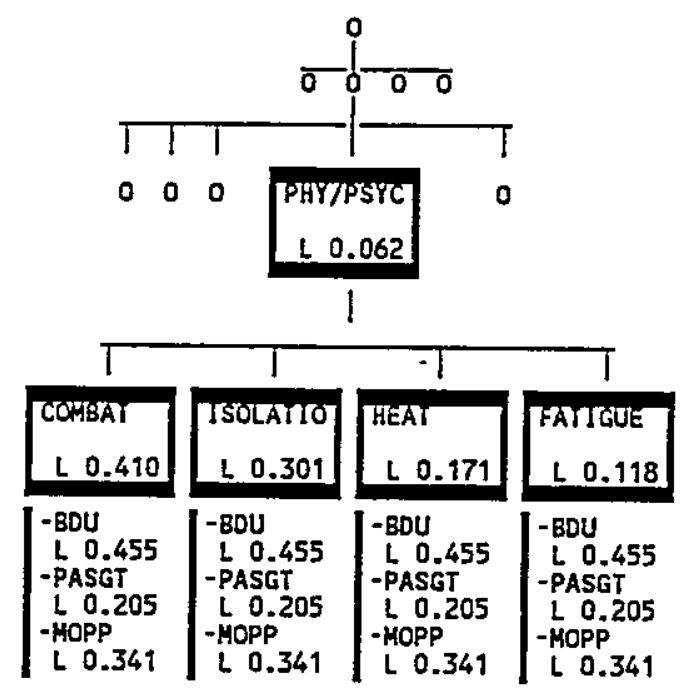

Figure 15: Hierarchy for Physiological and Psychological Factors. 


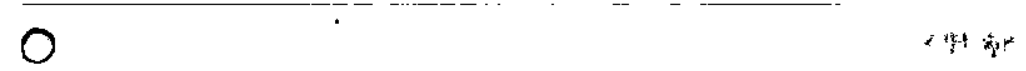

\subsection{Synergy Concerns:}

The Synergy factor evaluates the candidate SIPE modules with respect to the level of integration they achieve and in terms of how all the components fit together from the user's (individual soldier) perspective.
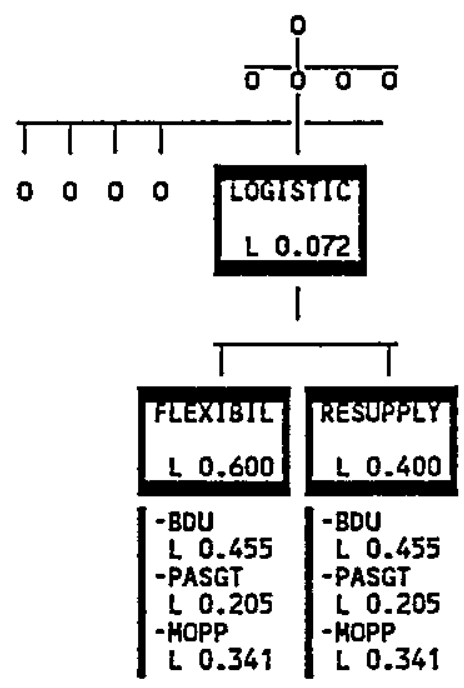

Figure 16: Hierarchy for Logistics Concerns.
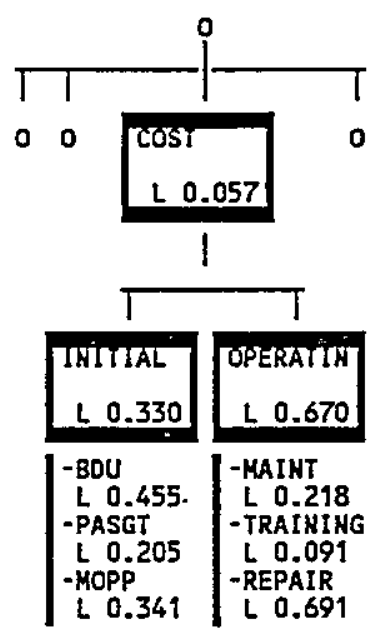

Figure 17: Hierarchy for Costs.

It is important to note that the model developed here will also provide guidance in collection of data for evaluation of SIPE modules.

\section{EVALUATION OF MODULES: AN OVERVIEW}

The BDU system does not provide any protection against ballistic weapons and chemical/biological weapons. It provides no protection against nuclear blast, but has some protection against radiation and thermal effects. The BDU provides protection against 
flame weapons and lasers. On the other hand, it has the least weight and cost among the three systems. The MOPP.system provides no protection against ballistic threats and against nuclear blast. It has strong protection against chemical/biological weapons and has some protection against laser weapons and nuclear radiation and thermal effects. It provides better protection than BDU against flame weapons. The MOPP system is capsulated system which is heavy and costs more than BDU. The PASGT is more expensive and slightly heavier than MOPP. Unlike the BDU and MOPP, it provides some ballistic protection. It also has some protection against chemical/biological and lasers. PASGT provides high protection against flame weapons and thermal nuclear radiation; it has some protection against nuclear radiation as well [see Reches (1988) for a review of the systems].

In Figure 18, we illustrate the priorities of the systems derived with respect to ballistic protection concerns. As expected PASGT is the most desirable system in this case. We note that priorities in Figure 6 show how the systems with respect to nuclear protection. Similarly, priorities in Figures 7, 8 and 9 of Section II provide comparison of the three systems with respect to protection against other weapon threats.

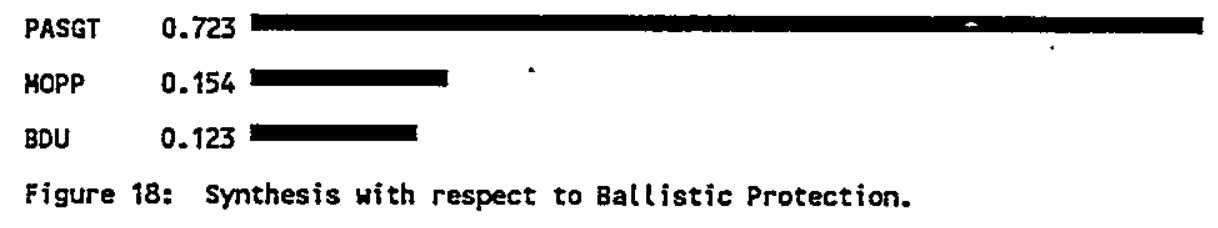

Figure 13 of Section II provides a comparison of the systems with respect to communication concerns. Similarly, Figures 14, 15, and 16 provides comparisons with respect to mobility, physiological/psychological and logistics concerns. The BDU system seems to be the most desirable with respect to performance criteria, but provides little protection for the soldier. In Figure 19, we illustrate the results of final synthesis with respect to the goal. Thus, our judgments indicate that the PASGT is the most desirable protection system among the currently available ones.

OVERALL IMCONSISTENCY INDEX $=0.06$

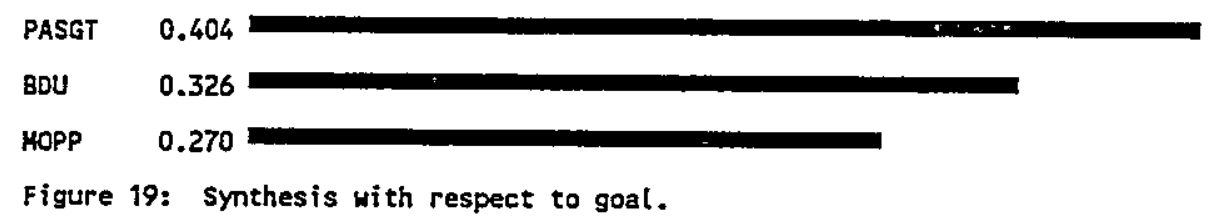

REFERENCES

Forman, E. H., Saaty, T. L., Selly, M. N. and Waldron, R. (1983). Expert Choice. Decision Support Software, Inc.

Reches, M. (1988). Dismounted Soldier Survivability. US Army Survivability Management Office. 


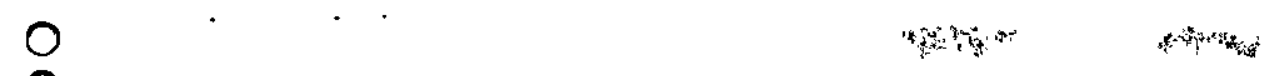

Saaty, T. L. (1980). The Analytic Hierarchy Process. McGraw-Hill.

Sampson, J. B., Herz, M. L. and Freitas, S. A. (1984). Program for the Development of an Integrated Combat Uniform System. Technical Report, NATICK/TN-84/010L, US Army Natick Research and Development Center.

Sampson, J. B., Tucker, D. W. and Ridgeway, D. C. (1989). Analysis of Combat Hazards for Balanced Protection. Technical Report, NATICK/TR-90/012L, US Army Natick Research and Development Center. 